\title{
Gas Absorption Center-Based Wavelength Calibration Technique in QEPAS System for SNR Improvement
}

\author{
Zongliang $\mathrm{WANG}^{1 *}$, Jun CHANG ${ }^{2}$, Qi LIU ${ }^{1}$, Cunwei $\mathrm{TIAN}^{1}$, and Qinduan ZHANG ${ }^{2}$ \\ ${ }^{1}$ School of Physics Science and Information Technology and Shandong Key Laboratory of Optical Communication \\ Science and Technology, Liaocheng University, Liaocheng 252000, China \\ ${ }^{2}$ School of Information Science and Engineering and Shandong Provincial Key Laboratory of Laser Technology and \\ Application, Shandong University, Jinan 250100, China \\ *Corresponding author: Zongliang WANG_ E-mail: wangzongliang@lcu.edu.cn
}

\begin{abstract}
A simple and effective wavelength calibration scheme is proposed in a quartz enhanced photoacoustic spectroscopy (QEPAS) system for trace gas detection. A reference gas cell is connected an InGaAs photodetector for detecting the absorption intensity peak caused by the gas to calibrate the gas absorption center using distributed feedback laser diode (DFB-LD) with sawtooth wave driver current. The gas absorption wavelength calibration and gas sensing operations are conducted at a special internal to eliminate the wavelength shift of DFB-LD caused by the ambient fluctuations. Compared with the conventional wavelength modulation spectroscopy (WMS), this method uses a lower lock-in amplifier bandwidth and averaging algorithm to improve signal noise ratio (SNR). Water vapor is chosen as a sample gas to evaluate its performance. In the experiments, the impact of sawtooth wave frequency and lock-in amplifier bandwidth on the harmonic signal is analyzed, and the wavelength-calibration technique-based system achieves a minimum detection limit (MDL) of 790ppbv and SNR with 13.4 improvement factor compared with the conventional WMS system.
\end{abstract}

Keywords: QEPAS; DFB-LD; wavelength calibration; fiber gas sensor

Citation: Zongliang WANG, Jun CHANG, Qi LIU, Cunwei TIAN, and Qinduan ZHANG, "Gas Absorption Center-Based Wavelength Calibration Technique in QEPAS System for SNR Improvement," Photonic Sensors, 2018, 8(4): 358-366.

\section{Introduction}

Trace gas detection plays an important role in series of fields, such as industrial production control $[1,2]$, mine safety monitoring $[3,4]$, combustion processes [5, 6], environmental monitoring [7] as well as explosive analysis, owing to the well-known advantages of resistance to electromagnetic interference, low cost, and portability. As a promising means for trace gas detection, infrared absorption spectroscopy (IRAS)-based sensors and photoacoustic spectroscopy (PAS)-based sensors have attracted considerable attention in recent years because of their effective selectivity, high sensitivity, and fast time response. However, as to IRAS sensors, a trace level of gas only leads to a minor attenuation of laser power that is hard to be differentiated by the photodetector, and optical power is easily saturated for photodetector by increasing laser power. These aspects mentioned above limit the signal noise ratio (SNR) of IRAS sensors. In comparison, PAS detects the gas concentration by detecting the

Received: 4 April 2018 / Revised: 16 May 2018

(C) The Author(s) 2018. This article is published with open access at Springerlink.com

DOI: $10.1007 / \mathrm{s} 13320-018-0502-0$

Article type: Regular 
laser-absorption induced acoustic signal using a microphone or quartz tuning fork (QTF). Without photodetector in the system, the PAS gas sensor can only achieve ultra-high sensitivity by eliminating the influence of background noise [8-10]. Quartz enhanced photoacoustic spectroscopy (QEPAS) is a variant of PAS, in which a commercially available high- $Q$ QTF is employed as a resonant acoustic transducer instead of the conventional wideband microphone. Due to the small size of the QTF, the QEPAS technique features an absorption detection module with dimensions of $1 \mathrm{~cm}-2 \mathrm{~cm}$ and uses the analysis of gas samples of a few $\mathrm{mm}^{3}$ in volume. As such, the QEPAS has been successfully applied to trace gases detection in numerous applications [11, 12].

With the development of science and technology, the more high SNR gas sensors are demanded in wide fields, the more methods are used to improve SNR. Wu et al. reported an innovative spectroscopic measurement technique that relies on the beat frequency signal between the QTF resonance frequency $f_{0}$ and the laser modulation frequency $f$ [13]. This technique can acquire the resonance frequency $f_{0}$ and $Q$-factor of the QTF as well as the trace-gas concentration information in a measurement time of about $30 \mathrm{~ms}$. It can be used for ultra-sensitive calibration-free trace-gas detection and fast spectral scan applications [13]. Sim et al. proposed an approach to match the resonant characteristics of a photoacoustic cell with that of a microphone in order to enhance the SNR in the photoacoustic sensor [14]. Using this approach, the signal-to-noise ratio is increased 3.5 -fold from the optimized to non-optimized cell in the photoacoustic spectroscopy system. Some novel QTFs and acoustic microresonator (AmR) are proposed to improve the SNR. Sampaolo et al. reported a QEPAS sensor for methanol detection by employing a novel QTF, which is specifically designed to enhance the QEPAS sensing performance in the terahertz spectral range [15]. A minimum detection limit (MDL) of $160 \mathrm{ppbv}$ in $30 \mathrm{~s}$ integration time represents a nearly one-order-of-magnitude improvement with respect to previous reports. Zheng et al. developed QEPAS with a single-tube AmR inserted between the prongs of a custom QTF. The single-tube on-beam QEPAS spectro-phone configuration improved the detection sensitivity by 2 orders of magnitude compared with a bare QTF [16].

Except for improving photoacoustic signal intensity, decreasing the noise is also effective for improving SNR in the QEPAS system. The gas absorption wavelength-stabilization technique is often used to suppress the wavelength drift of a semiconductor laser due to the fluctuation of laser temperature and injection current, using the lower filtering bandwidth and averaging algorithm applied to the QEPAS system for decreasing incoherent external acoustic noise and providing high system performance. Yanagawa et al. successfully developed a frequency locked technique to lock a DFB laser for ammonia sensing [17]. Gong et al. introduced a central-wavelength stabilized diode laser for QEPAS and successfully stabilized the central wavelength of the laser within $5 \mathrm{~s}$ [18]. The central-wavelength stabilized laser can enhance the detection SNR effectively by suppressing the incoherent noise in QEPAS. Wang et al. reported a QEPAS-based $\mathrm{H}_{2} \mathrm{O}$ sensor using a wavelengthstabilized DFB laser which is realized by the third harmonic and achieved an MDL of 1 ppmv [19]. Wang et al. introduced a wavelength stabilizationbased photoacoustic spectroscopy for methane detection, which achieved an MDL of $11.5 \mathrm{ppmv}$ at $10 \mathrm{~s}$ response time, and the third harmonic signal was used to generate the error signal for wavelength stabilization [20]. However, the conventional wavelength stabilization technique is very complex and difficult to be realized in practical applications because of the residual amplitude modulation.

In this paper, a simple and effective wavelength calibration scheme is proposed in the QEPAS 
system for the trace gas detection. A reference gas cell and InGaAs photodetector are used to detect the absorption intensity induced by the gas for calibrating the gas absorption center using distributed feedback laser diode (DFB-LD) with sawtooth wave driver current, regardless of the residual amplitude modulation. The driver current corresponding to gas absorption center is confirmed by the absorption intensity peak. The gas absorption wavelength calibration and gas sensing is conducted at a special interval to eliminate the wavelength shift of DFB-LD caused by the ambient fluctuations. This gas absorption center-based calibration method uses lower lock-in amplifier bandwidth and averaging algorithm to improve SNR. To validate the feasibility of these measures mentioned above, theoretical analysis and experiments are performed.

\section{Wavelength calibration method}

A wavelength calibration method is proposed to improve SNR using the absorption intensity peak. This method is described in details as follows:

(1) A sawtooth signal is used to drive the laser source, and the emitted light propagates through the reference gas cell, resulting in a decrease in laser intensity because of gas absorption. The laser passing through the reference gas cell is converted into electrical signals by the photodetector, and they are acquired by data acquisition card (DAQ).

(2) The acquired signals are transmitted to computer and then processed by fitting liner. The absorption intensity is achieved by the difference between acquired and fitted data.

(3) The wavelength driver current corresponding to gas absorption center is obtained by the one at the max absorption intensity. Then, the obtained current is set to the laser driver, and the gas sensing starts using the above constant driver current combined with a high frequency sine modulation signal to excited second harmonic photoacoustic signal by the gas absorption. The gas concentration is obtained by this photoacoustic signal.
The gas absorption wavelength calibration and gas sensing are conducted at a special interval to eliminate the wavelength shift of DFB-LD caused by the ambient fluctuations. The cycle of the wavelength calibration and gas sensing are $\mathrm{T} 1$ and T2, respectively. The wavelength calibration method and the laser driver signals distribution are shown in Figs. 1 and 2. In Fig. 1, the wavelength calibration method is clarified in three steps. Firstly, the DFB laser is driven by the sawtooth signal shown in solid line. Secondly, the laser transmitting the reference gas cell is acquired by the DAQ, and its fitting curve is shown in dashed line. Lastly, the absorption laser intensity induced by the gas is obtained by the subtraction, as shown in curve line. Then, the driver current corresponding to gas absorption center is obtained by the absorption intensity peak and is marked in the circle curve. As shown in Fig. 2, it can be seen that the DFB driver signal is only the sawtooth signal without the high frequency modulation signal in the wavelength calibration, by contrast, the DFB driver signal is direct current (DC) signal at the gas absorption center combined with the high frequency modulation signal in the gas sensing stage. Meanwhile, the gas absorption wavelength calibration and gas sensing operations are conducted at a special internal to eliminate the wavelength shift of DFB-LD caused by the ambient fluctuations.

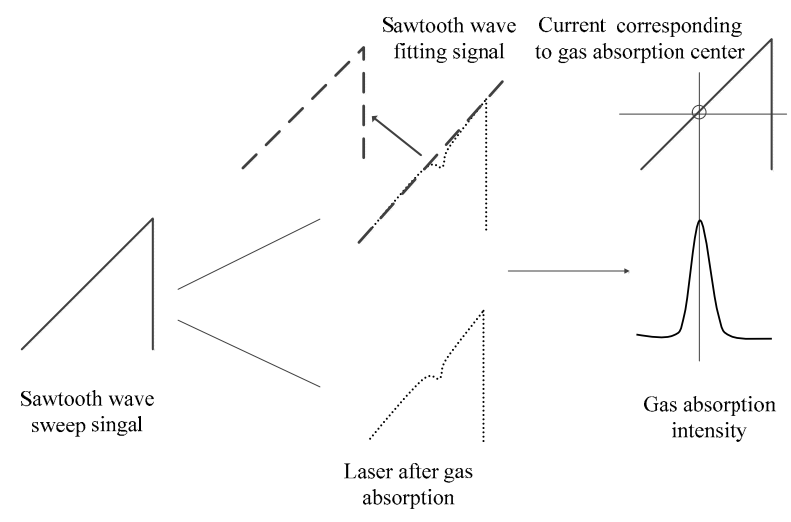

Fig. 1 Gas absorption center-based wavelength calibration method. 


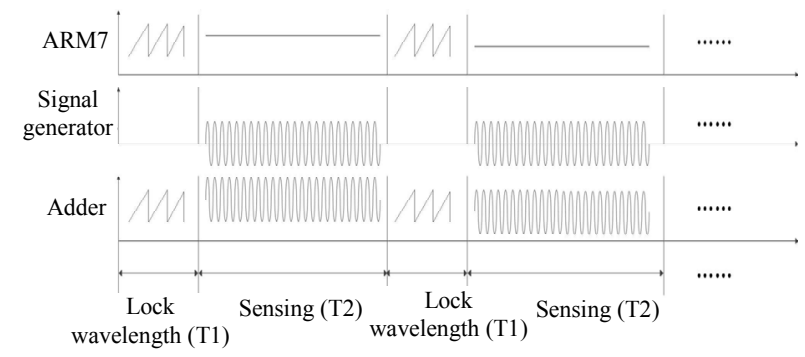

Fig. 2 Driver signals at gas absorption center calibration and sensing.

\section{Experiment}

\subsection{Experimental setup}

In experiment, water vapor was chosen as the sample gas to verify the gas absorption center-based wavelength calibration technique. The experimental setup was constructed as shown in Fig. 3. Water vapor has a strong absorption line at $1368.597 \mathrm{~nm}$ (7306.752 $\mathrm{cm}^{-1}$ ) according to HITRAN 2008 [19], so a 14-pin butterfly packaged DFB laser with a central wavelength of $1368.30 \mathrm{~nm}$ (Wuhan 69 Sensor Technology, CHINA) was utilized as the laser source. An ARM7 (LPC1758, NXP, Netherlands) and a signal generator (FY2300A, Feel Tech, China) were constructed as driving module to control the DFB laser. ARM7 was used to generate the sawtooth wave signal or the DC signal, and the signal generator was used to generate the two-channel high frequency signals with the same frequency for wavelength modulation and lock-in amplifier reference signal. The two signals generated by the ARM7 and signal generator were added as a laser final driver signal. In the gas absorption wavelength calibration process, the DFB laser used the sawtooth wave signal generated by ARM7 without the high frequency modulation signal as the driver signal. However, in the gas concentration measurement process, the DFB laser used the DC signal corresponding to gas absorption center combined with high frequency modulation signal as the driver signal. The custom-made transmission-type gas cell with two G-lens fiber collimators was adopted to detect the photoacoustic signal by a QTF. The resonant frequency of the QTF (DT-38, $32.768 \mathrm{kHz}, 12.5 \mathrm{pF})$ was calibrated as $f_{0}=$ $32.755 \mathrm{kHz}$ with a $3 \mathrm{~dB}$ bandwidth of $4 \mathrm{~Hz}$. The laser beam was collimated by the first collimator to pass through the gap between two fork prongs and was acquired by the other collimator connecting the reference gas cell. All the experimental measurements of target and reference gas cells were performed at the same condition with the pressure of 1 bar and room temperature. The laser beam was injected into the reference gas cell with $10-\mathrm{cm}$ length after propagating the QTF gas cell. Then, the laser was injected to photodetector through the reference gas cell to calibrate gas absorption center. The output electrical signals of photodetector were acquired and transferred to the computer by the DAQ for further processing without lock-in amplifier demodulation. In the computer, the absorption intensity peak induced by the gas was calculated by the output electrical signals of photodetector and its linear fitting curve using the subtraction. Meanwhile, the driver current corresponding to gas absorption center was obtained by the absorption intensity peak and transmitted to ARM7 for driving the laser. In the gas concentration measurement process, the harmonic current signals generated by the QTF were converted into voltage signals by the pre-amplifier and extracted by lock-in amplifier using the reference signal outputted by the signal generator. At last, the harmonic signals were acquired by DAQ and transmitted to the computer to calculate the gas concentration.

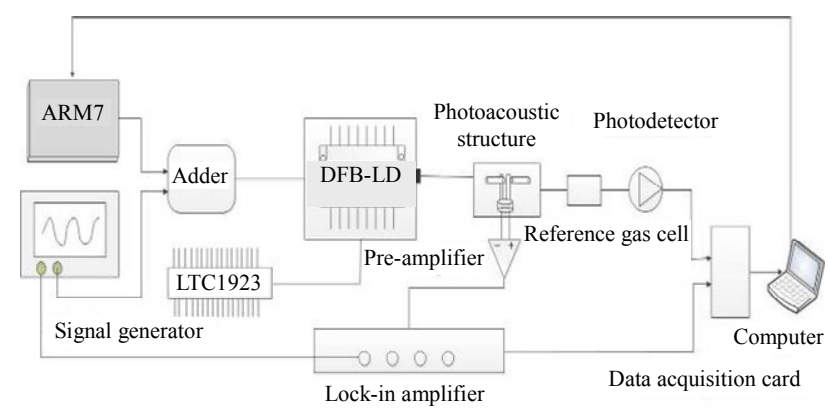

Fig. 3 Experimental setup. 


\subsection{Experimental result}

\subsubsection{Impact of sawtooth wave frequency to system performance}

In order to analyze the impact of sawtooth wave frequency on the system performance, the photoacoustic signals at different sawtooth wave frequencies among $0.1 \mathrm{~Hz}$ to $1.1 \mathrm{~Hz}$ were obtained at $450 \mathrm{ppm}$ gas concentration using experimental setup in Fig. 3. The experimental results are shown in Fig. 4. From Fig. 4, we can know that the photoacoustic signal decreases with an increase in the sawtooth wave frequency. This phenomenon was mainly caused by the lock-in amplifier bandwidth and the energy accumulative time of QTF. In the assumption that the frequencies of sawtooth wave and modulation signal were $\omega_{1}$ and $\omega_{0}$, respectively, then the frequency of the second harmonic signal was $2 \omega_{0}-\omega_{1}$ and $2 \omega_{0}+\omega_{1}$ and was no longer the only $2 \omega_{0}$. In this condition, the harmonic signal intensity was affected by the lock-in amplifier bandwidth. The harmonic signal could be demodulated perfectly if the lock-in amplifier bandwidth was greater than the $2 \omega_{1}$ bandwidth of harmonic signal, or else the harmonic signal will be decreased compared with the actual signal. Meanwhile, the sawtooth wave frequency was related to the energy accumulative time of QTF. In order to achieve the perfect second harmonic signal, the sawtooth wave cycle must be greater than the energy accumulative time of QTF, otherwise, the QTF could not respond to the harmonic signal.

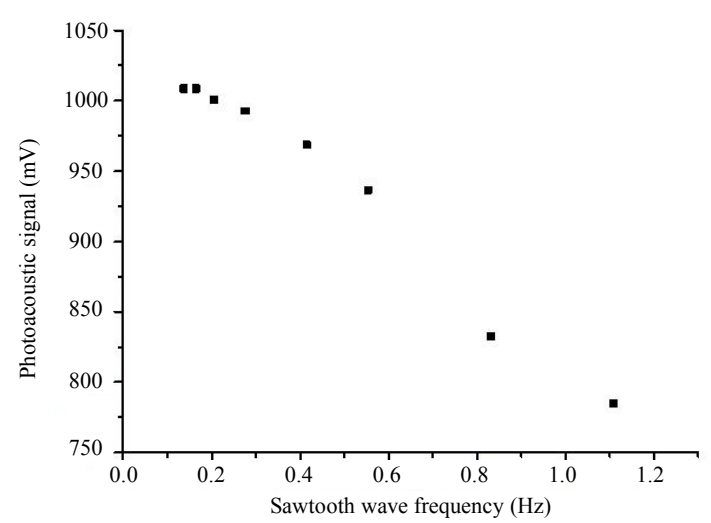

Fig. 4 Photoacoustic signal at different sawtooh wave frequencies.

\subsubsection{Impact of lock-in amplifier bandwidth on system performance}

The lock-in amplifier bandwidth also had great influence on photoacoustic signal intensity. The relation between the lock-in amplifier bandwidth and photoacoustic signal was implemented using the experimental setup in Fig. 3 at $450 \mathrm{ppm}$ gas concentration. The second harmonic photoacoustic signal of the conventional wavelength modulation spectroscopy (WMS) with $2.4 \mathrm{~s}$ sawtooh wave cycle and the wavelength calibration system were achieved at different lock-in amplifier bandwidths among $0.03 \mathrm{~Hz}$ to $35 \mathrm{~Hz}$, and the results were shown in Fig. 5. From Fig. 5(a), it can be seen that the photoacoustic signal intensity increases with the growth of the lock-in amplifier bandwidth and tends to be stable at last in the conventional WMS system. This is mainly because the photoacoustic signal not only has $2 \omega_{0}$ frequency ( $\omega_{0}$ modulation frequency) and but also has certain bandwidth caused by the sawtooh wave frequency $\omega_{1}$. In order to achieve the perfect photoacoustic signal, the lock-in amplifier bandwidth needs to be greater than the $2 \omega_{1}$ bandwidth of harmonic signal. As shown in Fig. 5(b), the lock-in amplifier bandwidth almost has no influence on photoacoustic signal intensity in the wave calibration system. This was mainly due to the narrow bandwidth photoacoustic signal in the wave calibration system. In the wave calibration system,

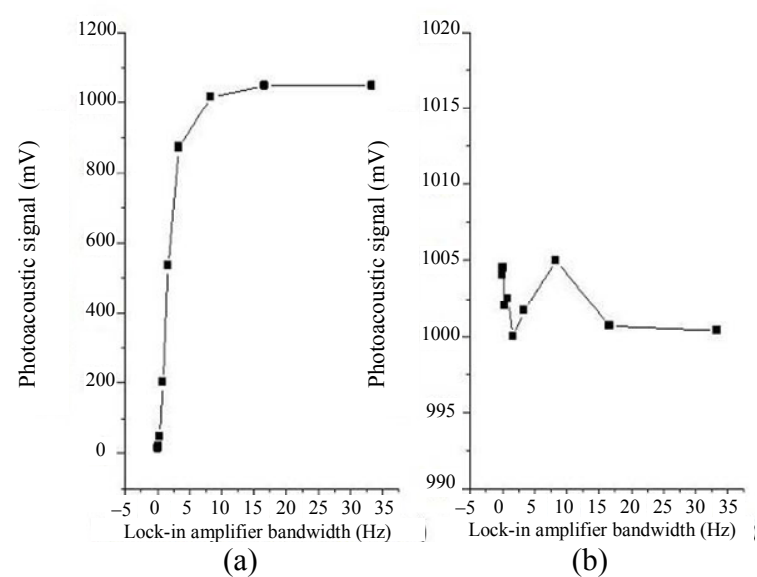

Fig. 5 Photoacoustic signal at different lock-in amplifier bandwidths: (a) photoacoustic signal with conventional WMS and (b) photoacoustic signal with new wavelength calibration technique. 
the DFB driver current was DC current combined with modulation signal and did not use the sawtooh wave, so the photoacoustic signal bandwidth was very narrow and the photoacoustic signal intensity was not affected by the lock-in amplifier bandwidth.

The noises at different amplifier bandwidths among $0.01 \mathrm{~Hz}$ to $35 \mathrm{~Hz}$ are shown in Fig. 6. It can be observed that the noise decreases with the reduction of the lock-in amplifier bandwidth. Comparing with the conventional WMS system, the wavelength calibration system can improve SNR by a narrow lock-in amplifier bandwidth.

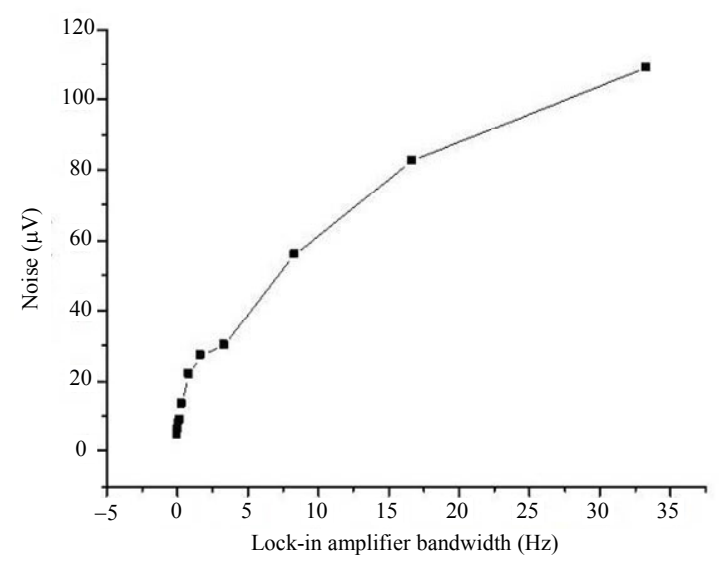

Fig. 6 Signal noise at different lock-in amplifier bandwidths.

\subsubsection{Wavelength calibration technique compared with wavelength stabilization scheme}

The comparative experiment between the wavelength stabilization scheme and the wavelength calibration technique proposed in this paper was implemented to verify the wavelength calibration effectiveness in the proposed method. In the wavelength stabilization scheme, the laser wavelength was obtained by the absorption intensity peak and then kept constant. In the wavelength calibration technique, the laser wavelength was calibrated discontinuously by the absorption intensity peak as shown above. According to the wavelength calibration technique mentioned above, the laser intensity detected by the photodetector after the reference gas cell is shown in Fig. 7. In Fig. 7, the dotted line represents the laser intensity through the reference cell, and the solid line is the linear fitting line for the laser intensity. The absorption intensity was obtained by the subtraction between the solid line and the dotted line in Fig. 7 and its Lorentzian fitting curve are plotted by the dotted and solid curve in Fig. 8(a). The driver voltage at the peak of the fitting curve is regarded as the one of the gas absorption center, as shown in Fig. 8(b).

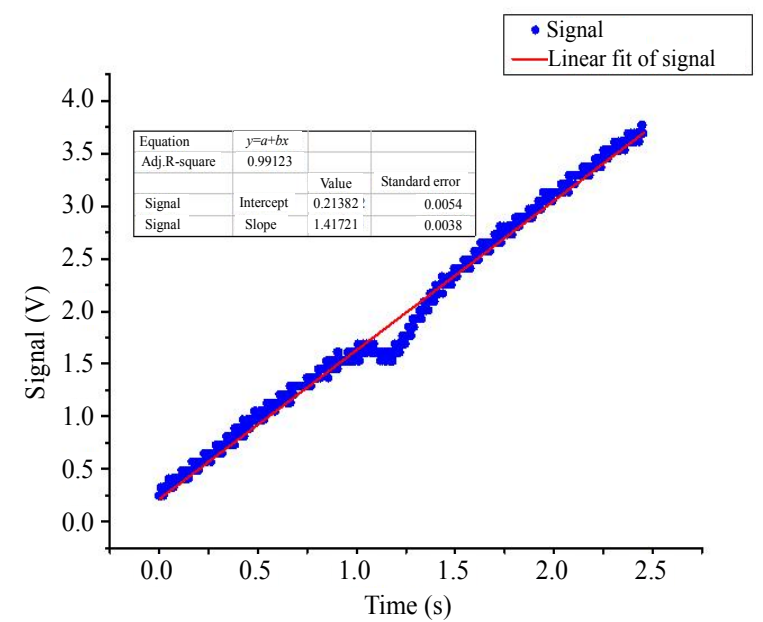

Fig. 7 Laser intensity through the reference gas cell.

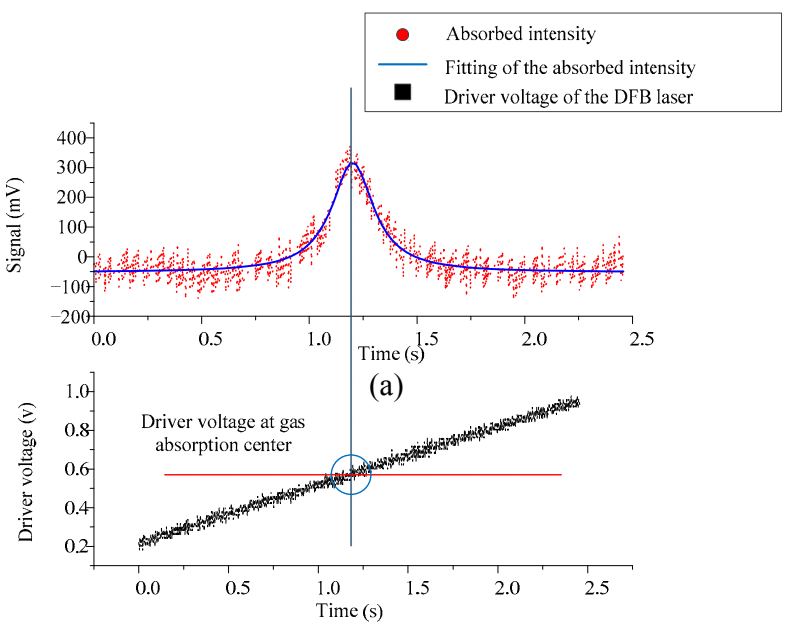

(b)

Fig. 8 Absorption laser intensity and driver voltage at gas absorption center: (a) the absorption laser intensity of the reference gas cell and (b) the driver voltage of DFB laser.

The comparative experimental results at 360 ppm are shown in Fig. 9. From Fig. 9, it can be observed that the photoacoustic signal is about $908 \mathrm{mV}$ with $20 \mathrm{mV}$ fluctuation in wavelength stabilization scheme, nevertheless, the wavelength calibration technique is about $908 \mathrm{mV}$ with $6 \mathrm{mV}$ fluctuation. It is mainly because that the wavelength calibration technique can eliminate the wavelength 
shift of DFB-LD caused by the ambient fluctuations, such as temperature and pressure.

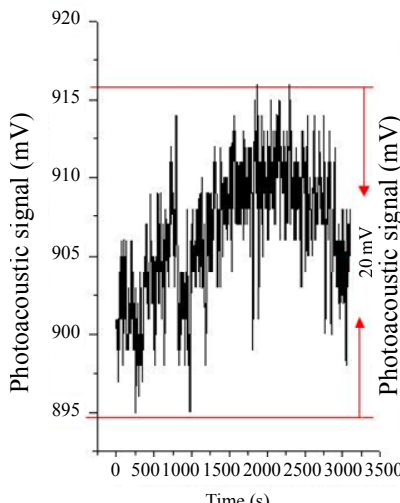

(a)

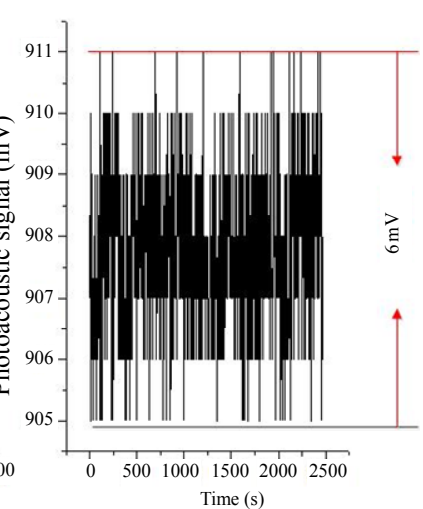

(b)
Fig. 9 Photoacoustic signal in: (a) wavelength stabilization scheme and (b) calibration technique.

\subsubsection{Wavelength calibration technique compared with the conventional WMS system}

In order to analyze the SNR improvement of this wavelength calibration technique, the comparative experiment between the conventional WMS system and the wavelength calibration technique proposed in this paper is implemented using the experimental setup shown in Fig. 3. In the conventional WMS system, the reference gas cell and the photodetector in Fig. 3 are not used because they were no longer used to calibrate in the gas absorption center. In this experiment, the experimental parameters in the wavelength calibration technique and conventional WMS system are the same except for the lock-in amplifier bandwidth. In order to achieve the similar order of photoacoustic signal amplitude, the lock-in amplifier bandwidth in the wavelength calibration technique was $0.08 \mathrm{~Hz}$, but for the conventional WMS system it was $8 \mathrm{~Hz}$. Or else the photoacoustic signal amplitude would be obviously different in the two systems because of the impact of sawtooh wave frequency. The scanning cycle of $2.4 \mathrm{~s}$ was chosen in the conventional WMS system, so there would be only one data point of second harmonic signal acquired per $2.4 \mathrm{~s}$ in this system. However, in our wavelength calibration system, 6000 data points were collected and averaged by DAQ in $2.4 \mathrm{~s}$ for a lower noise level. The noise in the wavelength calibration system could be degraded by the numerical averaging algorithm. A dew-point generator was used to produce $600 \mathrm{ppm}$ water vapor samples for this experiment. The photoacoustic signal in the wavelength calibration scheme and conventional WMS system were collected as shown in Fig. 10. As shown in Fig. 10(a), the photoacoustic signal in the conventional WMS system is about $1192 \mathrm{mV}$ with $21.12 \mathrm{mV}$ noise. In contrast, the photoacoustic signal in wavelength calibration technique is about $1207.4 \mathrm{mV}$ with $1.595 \mathrm{mV}$ noise, and the MDL is about $790 \mathrm{ppbv}$ in Fig. 10(b). The amplitude of photoacoustic signal in the conventional WMS system is lower than that in the wavelength calibration technique, mainly because the time of photoacoustic energy accumulation limits the photoacoustic signal. Moreover, the noise in the wavelength calibration technique is reduced by the numerical averaging algorithm. By calculation, the SNR in the wavelength calibration technique is increased 13.4 times compared with the conventional WMS system.

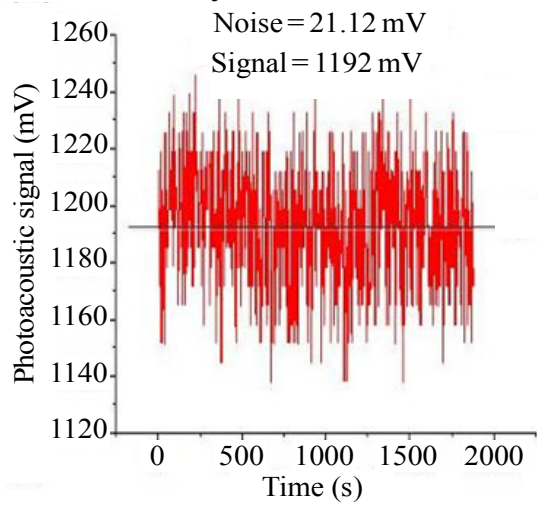

(a)

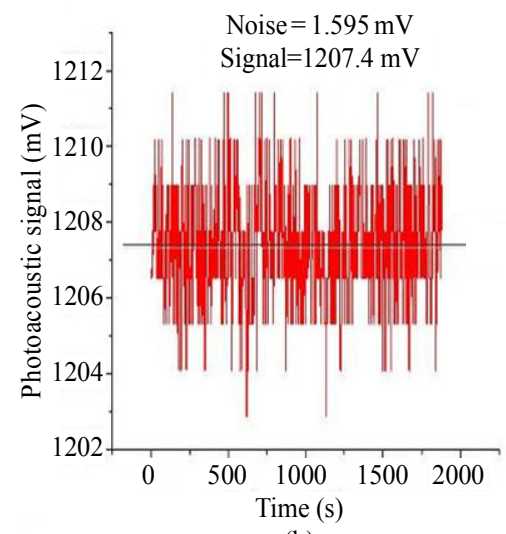

(b)

Fig. 10 Photoacoustic signal in: (a) conventional WMS system and (b) wavelength calibration technique. 


\section{Discussion}

The gas absorption wavelength-stabilization technique is often used to improve the QEPAS system performance by using the lower filtering bandwidth and averaging algorithm. The harmonic signals are usually used to stabilize the gas absorption center in the wavelength stabilization technique. However, the harmonic signals at the gas absorption center are hard to determine because the harmonic signals are changed by the residual amplitude modulation compared with theoretical harmonic signals, and the harmonic signals are influenced by the gas concentration. Therefore, the conventional gas absorption wavelengthstabilization technique is very complex and difficult to be realized in practical applications. In this paper, a simple and effective wavelength calibration technique is proposed in the QEPAS system by detecting the absorption intensity peak induced by the gas for improving SNR regardless of the residual amplitude modulation. In this method, the absorption intensity peak is detected to calibrate the gas absorption center using DFB-LD with sawtooth wave driver current, and then the wavelength of the diode laser is locked to the target gas absorption center to detect gas concentration with high frequency sine modulation signals.

\section{Conclusions}

In this paper, a simple and effective wavelength calibration technique is proposed to improve SNR in the QEPAS system for trace gas detection regardless of the residual amplitude modulation. In this method, a reference cell connected to an InGaAs photodetector is adopted to detect the absorption intensity peak to calibrate the gas absorption center wavelength using DFB-LD with sawtooth wave driver current, and then the wavelength of the diode laser is locked to the target gas absorption center to detect gas concentration with high frequency sine modulation signals. The gas absorption wavelength calibration and gas sensing are conducted at a special interval to eliminate the wavelength shift of DFB-LD caused by the ambient fluctuations. Compared with the conventional WMS system, this method ensures a QTF output maximum signal by using the lower lock-in amplifier bandwidth and the averaging algorithm to improve detection limit and SNR effectively. Moreover, the impact of sawtooth wave frequency and lock-in amplifier bandwidth on the harmonic signal are analyzed in the paper. The lower frequency sawtooth wave can enhance the photoacoustic signal due to the enough time for photoacoustic energy accumulation, and the lower lock-in amplifier bandwidth can decrease noise. In the water vapor sensing experiments, the novel system achieves an MDL of $790 \mathrm{ppbv}$ and SNR with 13.4 improvement factor compared with the conventional WMS system. This method contributes to the foundation for future research aiming at improving the SNR in QEPAS system.

\section{Acknowledgment}

This work was supported by Research Fund for the Doctoral Program of Liaocheng University (Grant No. 318051543) and the National Natural Science Foundation of China (Grant No. 61475085).

Open Access This article is distributed under the terms of the Creative Commons Attribution 4.0 International License (http://creativecommons.org/licenses/by/4.0/), which permits unrestricted use, distribution, and reproduction in any medium, provided you give appropriate credit to the original author(s) and the source, provide a link to the Creative Commons license, and indicate if changes were made.

\section{References}

[1] S. Rasi, A. Veijanen, and J. Rintala, "Trace compounds of biogas from different biogas production plants," Energy, 2007, 32(8): 1375-1380.

[2] X. Chen, J. Chang, F. P. Wang, Z. L. Wang, W. Wei, Y. Y. Liu, et al., "A portable analog lock-in amplifier for accurate phase measurement and application in high-precision optical oxygen concentration detection," Photonic Sensors, 2017, 7(1): 27-36.

[3] X. G. Niu, X. Huang, Z. Zhao, Y. H. Zhang, C. C. Huang, and L. Cui, "The design and evaluation of a 
wireless sensor network for mine safety monitoring," in Proceeding of IEEE Global Telecommunications Conference, Washington, DC, USA, 2007, pp. 1291-1295.

[4] J. P. Sun, "Mine safety monitoring and control technology and system," Coal Science and Technology, 2010, 38(10): 1-4.

[5] M. Allen, "Diode laser absorption sensors for gas-dynamic and combustion flows," Measurement Science and Technology, 1998, 9(4): 545-562.

[6] Y. Liu, E. Koep, and M. L. Liu, "A highly sensitive and fast-responding $\mathrm{SnO}_{2}$ sensor fabricated by combustion chemical vapor deposition," Chemistry of Materials, 2005, 17(15): 3997-4000.

[7] A. R. Brandt, G. A. Heath, E. A. Kort, F. O'Sullivan, G. Pétron, S. M. Jordaan, et al., "Methane leaks from North American natural gas systems," Science, 2014, 343(6172): 733-735.

[8] J. Wojtas, A. Gluszek, A. Hudzikowski, and F. K. Tittel, "Mid-infrared trace gas sensor technology based on intracavity quartz-enhanced photoacoustic spectroscopy," Sensors, 2017, 17(3): 513-1-513-9.

[9] Y. F. Ma, Y. He, C. Chen, X. Yu, J. B. Zhang, J. B. Peng, et al., "Planar laser-based QEPAS trace gas sensor," Sensors, 2016, 16(7): 989-1-989-7.

[10] Q. D. Zhang, J. Chang, Q. Wang, Z. L. Wang, F. P. Wang, and Z. G. Qin, "Acousto-optic Q-switched fiber laser-based intra-cavity photoacoustic spectroscopy for trace gas detection," Sensors, 2018, 18(1): 42-1-42-8.

[11] Z. Wang, J. Geng, and W. Ren, "Quartz-enhanced photoacoustic spectroscopy (QEPAS) detection of the $v 7$ band of ethylene at low pressure with $\mathrm{CO}_{2}$ interference analysis," Applied Spectroscopy, 2017, 71(8): 1834-1841.

[12] T. N. Ba, M. Triki, G. Desbrosses, and A. Vicet, "Quartz-enhanced photoacoustic spectroscopy sensor for ethylene detection with a $3.32 \mu \mathrm{m}$ distributed feedback laser diode," Review of Scientific Instruments, 2015, 86(2): 023111-1023111-5.

[13] H. Wu, L. Dong, H. Zheng, Y. J. Yu, W. G. Ma, L. Zhang, et al., "Beat frequency quartz-enhanced photoacoustic spectroscopy for fast and calibration-free continuous trace-gas monitoring," Nature Communications, 2017, 8: 15331-1-15331-8.

[14] J. Y. Sim, C. G. Ahn, C. Huh, K. H. Chung, E. J. Jeong, and B. K. Kim, "Synergetic resonance matching of a microphone and a photoacoustic cell," Sensors, 2017, 17(4): 804-1-804-10.

[15] A. Sampaolo, P. Patimisco, M. Giglio, M. S. Vitiello, H. E. Beere, D. A. Ritchie, et al., "Improved tuning fork for terahertz quartz-enhanced photoacoustic spectroscopy," Sensors, 2016, 16(4): 439-1-439-8.

[16] H. D. Zheng, L. Dong, A. Sampaolo, H. P Wu, P. Patimisco, X. K. Yin, et al., "Single-tube on-beam quartz-enhanced photoacoustic spectroscopy," Optics Letters, 2016, 41(5): 978-981.

[17] T. Yanagawa, S. Saito, and Y. Yamamoto, "Frequency stabilization of $1.5 \mu \mathrm{m}$ InGaAsP distributed feedback laser to $\mathrm{NH}_{3}$ absorption lines," Applied Physics Letters, 1984, 45(8): 826-828.

[18] P. Gong, L. Xie, X. Q. Qi, and R. Wang, "A QEPAS-based central wavelength stabilized diode laser for gas sensing," IEEE Photonics Technology Letters, 2015, 27(5): 545-548.

[19] G. S. Wang, H. M. Yi, T. D. Cai, L. Wang, T. Tan, W. J. Zhang, et al., "Research on the real-time measurement system based on QEPAS," Acta Physica Sinica, 2012, 61(12): 120701-1-120701-8.

[20] Q. Wang, Z. Wang, and W. Ren, "Wavelengthstabilization-based photoacoustic spectroscopy for methane detection," Measurement Science and Technology, 2017, 28(6): 065102-1-065102-8. 